\title{
A Lone Attosecond Electron Pulse
}

\section{Researchers generate isolated, high-energy electron pulses that promise ultrafast electron imaging and more.}

\author{
By Marric Stephens
}

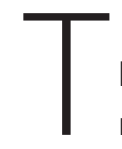

he ability to generate attosecond pulses of light has revolutionized research into ultrafast processes, revealing, for example, the motion of electrons in atoms. Attosecond electron pulses could be as revolutionary as their optical counterparts, but so far, scientists have only been able to produce them in trains hundreds of pulses long. Now, Yuya Morimoto at the Ludwig Maximilian University of Munich and Peter Baum at the University of Konstanz, Germany, have found a way to isolate a single attosecond-scale electron pulse [1]. This control promises to advance ultrafast imaging by giving it the spatial resolution of electron microscopy. Morimoto and Baum say that their technique could also have applications in chip-based particle accelerators and in quantum information processing technologies.

The duo's experiments involved two parallel, 20-nm-thick, metal-coated screens arranged sequentially, $12 \mathrm{~mm}$ apart. These screens were transparent to electrons but reflective to light. The researchers fired a beam of electrons through the

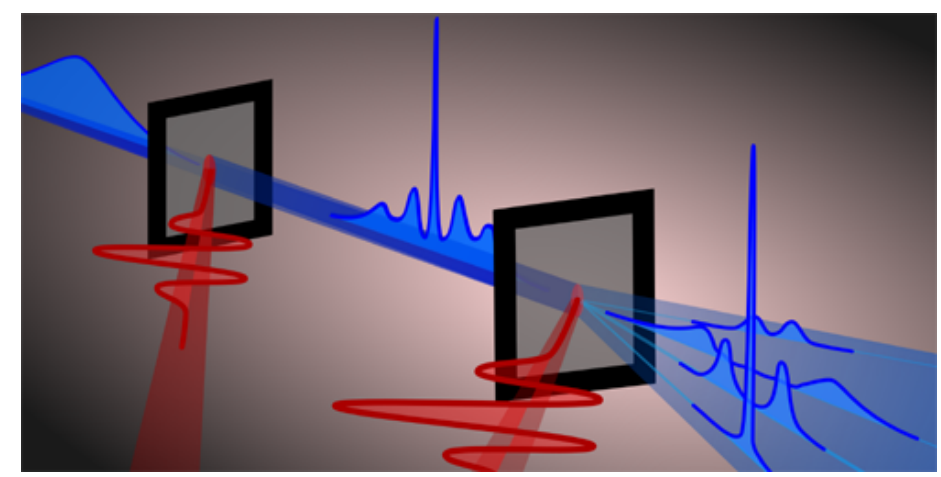

Credit: Y. Morimoto/Ludwig Maximilian University of Munich front of the two screens while shining THz-frequency, single-cycle light pulses onto the back of each.

Electrons passing through the screens emerged suddenly into the light's electric field. At the first screen, this field accelerated the electrons by an amount that depended on the phase of the light, shaping the beam into a sequence of bunches. At the second screen, the varying phase of the electric field deflected the path of the electrons up or down, spatially separating the bunches. This separation let the researchers isolate individual electron pulses with durations as short as 800 attoseconds-a length comparable to those achieved previously with electron-pulse trains.

Marric Stephens is a Corresponding Editor for Physics based in Bristol, UK.

\section{REFERENCES}

1. Y. Morimoto and P. Baum, "Single-cycle optical control of beam electrons," Phys. Rev. Lett. 125, 193202 (2020). 